\title{
RECURSOS PARA LAS BUENAS PRÁCTICAS Y LA EVALUACIÓN FORMATIVA (EDUCACIÓN INFANTIL Y PRIMARIA)
}

Resources for good practices and formative assessment (Pre-school and Primary

\section{Education)}

Recursos para boas práticas e avaliação formativa (Educação Pré-escolar e Primária)

\section{Pablo Casado Berrocal (1)}

\section{Óscar Manuel Casado Berrocal (2)}

(1) CEIP Villa y Tierra, Saldaña (Palencia), España. Teléfono: +34 660821826. Correo electrónico: pablo.casber@educa.jcyl.es

(2) CEIP Ponce de León (León), España. Teléfono: +34 646920522 Correo electrónico: oscar.casado.berrocal@gmail.com

\begin{abstract}
Resumen
Las herramientas digitales suponen hoy en día una gran ayuda a la hora de desarrollar el proceso de evaluación del aprendizaje. Los docentes deben conocer y saber utilizar las últimas novedades en este sector. Para ello, en el presente taller se presentarán algunas de las herramientas y aplicaciones digitales más interesantes para utilizar en las etapas de Educación infantil y Primaria. Se pretende proporcionar estrategias y recursos para la generación de materiales por parte del alumnado, el uso de sistemas compartidos de información o la gestión del aula en contextos de interacción con las familias. Del mismo modo, también se presentarán alternativas al tradicional examen escrito así como sistemas digitales para la gestión de las calificaciones de los alumnos.

Palabras clave: Buenas prácticas; evaluación formativa; evaluación compartida; Infantil; Primaria; apps
\end{abstract}

\begin{abstract}
Digital tools nowadays are a great help in developing the learning assessment process. Teachers must know and know how to use the latest developments in this sector. To this end, this workshop will present some of the most interesting digital tools and applications to use in the stages of Nursery School and Primary Education. It aims to Recursos para las buenas prácticas y la evaluación formativa (Educación Infantil y Primaria)
\end{abstract}


provide strategies and resources for the creation of materials by students, the use of shared information systems or classroom management in contexts of interaction with families. Likewise, alternatives to the traditional written exam will also be presented as well as digital systems for the management of the students' grades.

Keywords: Good practices; formative assessment; shared assessment; Nursery School; Primary Education; apps

\section{Resumo}

As ferramentas digitais representam uma grande ajuda no desenvolvimento do processo de avaliação da aprendizagem. Os professores devem conhecer e ser capaz de usar as últimas novidades neste sector. Para fazer isso, algumas das ferramentas e aplicações digitais mais interessantes para usar em etapas da Educação Básica serão apresentadas. Visa fornecer estratégias e recursos para a geração de materiais pelos alunos, o uso de sistemas de informação compartilhados ou gestão de sala de aula em contextos de interação com as famílias. Da mesma forma, também serão apresentadas alternativas para o tradicional exame escrito e sistemas digitais para gerenciar notas dos alunos.

Palavras-chave: Boas práticas; avaliação formativa; co-avaliação; Educação Básica, apps

\section{Evaluación formativa y recursos didácticos para la práctica educativa}

En los últimos años asistimos a una situación socioeducativa protagonizada por los constantes esfuerzos que se realizan para mejorar los procesos de aprendizaje. Como parte fundamental de estos procesos, la evaluación y la forma en la que esta se lleva a cabo también ha supuesto objeto de reflexión.

En líneas generales, evaluar consiste en realizar un seguimiento a lo largo del proceso de aprendizaje que permita obtener información acerca de cómo se está llevando a cabo, con el fin de reajustar la intervención educativa, de acuerdo con los datos obtenidos (MEC, 1996). Para reajustar de forma exitosa esa intervención, la participación del alumno en la evaluación es decisiva. Por ello, los modelos relacionados con la evaluación formativa y compartida (López-Pastor \& Pérez-Pueyo, 2017), cuyo principal objetivo es mejorar y reconducir el proceso de aprendizaje mediante la toma de decisiones dialogada, se encuentran en pleno auge. Estos modelos, 
por su parte, requieren del uso de instrumentos claros y otros recursos didácticos de calidad, elegidos de forma coherente respecto al resto de intenciones educativas.

Y es que, en el diseño de la práctica educativa, la mirada del docente se centra de forma inevitable en los recursos que tiene a su disposición y, en consecuencia, en la forma que estos van a ser utilizados por él y por sus alumnos.

Aunque habitualmente los centros parten de unas determinadas condiciones que, en muchos casos, no está en manos del profesorado modificar, dentro de estos márgenes reales queda suficiente juego para poder tomar decisiones de gran relevancia. Por ello, la regla de oro tácita por la que debemos regirnos a la hora de delimitar los recursos didácticos que emplearemos en el conjunto de procesos asociados a nuestra práctica educativa (incluida, la evaluación) es: debemos aprovechar al máximo los recursos que tenemos a nuestro alcance; y uno de los recursos protagonistas en los últimos años son las Tecnologías de la Información y la Comunicación (TIC).

Son muchos los autores que han escrito evidenciando la incidencia que los recursos y herramientas digitales pueden tener sobre la mejora del aprendizaje de los alumnos (Bigun \& Kenway, 1998; Cabero, 1998; Marqués, 2003; Lozano, 2011; Moya, 2013; Belloch, 2014; etc.). No en vano, cada vez son más las empresas y asociaciones que intentan diseñar herramientas y aplicaciones específicas para aplicar en entornos educativos, lo cual es una evidencia más de la gran demanda que existe en este ámbito. Ahora bien, de nada sirve disponer de recursos variados si el profesorado no sabe cómo aprovecharlos y utilizarlos.

Con este objetivo, en este taller se abordarán reflexiones de interés relacionadas con el uso de las TIC en los procesos de aprendizaje, abarcando momentos que van desde la selección y presentación de herramientas digitales hasta la resolución de conflictos habituales en el día a día del aula; cuestiones todas ellas necesarias para contribuir a la incorporación efectiva de las TIC en los procesos educativos (LOE, 2006: Preámbulo).

\section{Contextualización del taller}

Los contenidos que se abordan en el desarrollo de este taller están centrados en las etapas educativas de Educación Infantil y Educación Primaria por tratarse de elementos interés para los periodos de edad a los que se refieren ambas etapas. 
Aunque la mayoría de los contenidos se tratan de forma general, en ocasiones será necesario contextualizar el uso de ciertas técnicas y herramientas para cada etapa educativa.

Asimismo, se pretende desarrollar el taller a través de una dinámica dialogante en forma de coloquio que permita tener en cuenta las aportaciones de los asistentes con la intención de contextualizar cada una de las propuestas que se plantean a la diversidad de realidades educativas que rodean el entorno de cada centro escolar.

\section{Descripción del taller}

El taller ofrece a los asistentes un modelo pautado para la incorporación efectiva de las TIC a su práctica educativa. Durante su desarrollo, se abordan cuestiones asociadas al origen del uso de las TIC en Educación y su evolución hacia las Tecnologías del Aprendizaje y el Conocimiento (TAC) y las Tecnologías del Empoderamiento y la Participación (TEP) (Reig, 2011) para, posteriormente, realizar una puesta en común acerca de los procesos más eficaces para la selección de herramientas digitales y organización de estas en los Entornos Personales de Aprendizaje de alumnos y docentes.

La parte central del taller pretende ofrecer alternativas, tanto a los docentes como a los alumnos, sobre las opciones que se vinculan con las nuevas tecnologías de la información y la comunicación vinculadas al aprendizaje. Cada docente analizará el contexto educativo y tecnológico en el que desarrolla su función profesional (características del alumnado, capacidades y/o competencias a desarrollar en el alumnado, recursos disponibles, entre otros), para decidir entre algunas de las opciones siguientes:

- Generación de recursos a través de herramientas como Rubistar, Playposit, Educaplay, TypeA4, Blockposter, que permiten obtener resultados de calidad y un ahorro considerable en el tiempo dedicado a esta tarea.

- Producciones del alumnado mediante el uso de herramientas como Scratch, Padlet, Meeting words, Office365, G-Suite o StoryStartes que facilitan la generación de productos digitales (documentos, presentaciones, reportajes, actividades, videojuegos, etc.) mediante sencillos procesos vinculados, en la mayoría de los casos, con la colaboración en tiempo real entre el alumnado. 
- Desarrollo de la competencia lingüística de manera lúdica y contextualizada a través de herramientas como Morfo, MadLipz o LyricsTrainning (esta última para el trabajo de los idiomas).

- Evaluación formativa a través de herramientas como Quizizz, Plickers o Kahoot que no solo facilitan el desarrollo de los procesos de evaluación sino que además aumenta la posibilidad de generar actitudes positivas en el alumnado hacia ellos.

- Gestión de las calificaciones y demás registros de los alumnos a través de diarios digitales para el profesorado, como iDoceo o Additio.

- Mejora en la gestión de aula y participación familiar a través de plataformas como Class Dojo, ClassCraft o Edmodo, opciones que permiten externalizar determinados procesos que ocurren en el aula y facilitar la interacción entre los miembros de la comunidad educativa.

- Resolución de problemas TIC básicos en el aula y otras muchas opciones mediante el uso de ciertas estrategias técnicas sencillas y algunos recursos y aplicaciones offline para dispositivos móviles (tablets, móviles, portátiles, etc.), recomendables para aquellos contextos escolares que disponen de una conexión a Internet deficitaria.

\section{Reflexiones finales}

Si queremos extraer el máximo potencial al proceso de evaluación es preciso conocer más de un sistema o herramienta para extraer información relevante sobre el aprendizaje de los alumnos. Las TIC nos abren la puerta a un mundo nuevo lleno de posibilidades que es necesario conocer y saber manejar. A veces es su estructura, a veces su apariencia y otras veces simplemente, el componente lúdico. Pero las herramientas digitales que los docentes actuales tienen a su alcance funcionan y motivan al alumnado, haciendo casi obligatorio desempeñar procesos integrados de evaluación que vayan más allá del tradicional examen escrito. La evaluación, por tanto, no debe ser considerada como algo finalista, sino procesual, integrado en el día a día y en la cotidianeidad de la vida escolar.

\section{Referencias}

Adell, J. (1997). Tendencias en educación en la sociedad de las tecnologías de la información. En Revista Electrónica de Tecnología Educativa, 1(7). Universidad de las Islas Baleares. 
Belloch, C. (2014). Las TIC en el aprendizaje. Unidad de Tecnología Educativa. Universidad de Valencia.

Bigun, C. \& Kenway, J. (1998). New information technologies and ambiguous future of schooling. Some posible scenarios. En A.Hargreaves et al (Ed.), International Handbook of Educational Change. Dordrecht: Kluwer Academic Publishers.

Cabero, J. (1998). Impacto de las nuevas tecnologías de la información y la comunicación en las organizaciones educativas. En M. Lorenzo y otros (coords.), Enfoques en la organización y dirección de instituciones educativas formales y no formales. Granada: Grupo Editorial Universitario.

Ley Orgánica 2/2006, de 3 de mayo, de Educación en su texto consolidado por la Ley Orgánica 8/2013, de 9 de diciembre, para la Mejora de la Calidad Educativa (BOE de 29 de julio de 2015).

López-Pastor, V. M. \& Pérez-Pueyo, A. (Coords.) (2017). Evaluación formativa y compartida en educación: experiencias de éxito en todas las etapas educativas. León: Universidad de León. Recuperado de https://buleria.unileon.es/handle/10612/5999

López-Pastor, V.M. (Coord.) (2006). La Evaluación en Educación Física. Revisión de los modelos tradicionales y planteamiento de una alternativa: la evaluación formativa y compartida. Buenos Aires: Miñó y Dávila

Lozano, R. (2011). De las TIC a las TAC: tecnologías del aprendizaje y del conocimiento. Anuario ThinkEPI.

Marqués, P. (2003). Las TIC en la enseñanza obligatoria. Barcelona: CissPraxis.

Moya, M. (2013). De las TIC a las TAC: la importancia de crear contenidos educativos digitales. En Revista Didáctica, Innovación y Multimedia, 1(27). Recuperado de http://www.pangea.org/dim/revista27.htm

Reig, D. (2011). TIC, TAC, TEP y el 15 de octubre. Recuperado de http://www.dreig.eu/caparazon/2011/10/11/tic-tac-tep/ 